Research Paper

\title{
MicroRNA-216b targets HK2 to potentiate autophagy and apoptosis of breast cancer cells via the mTOR signaling pathway
}

\author{
Ting Liu1, ${ }^{\bowtie}$, Ping Ye3, Yuanyuan Ye², Baosan Han² \\ 1. The Affiliated Hospital of Qingdao University, Qingdao 266000, P.R. China. \\ 2. Department of General Surgery, Xinhua Hospital, School of Medicine, Shanghai Jiao Tong University, Shanghai 200092, P.R. China \\ 3. School of Medical Instrument and Food Engineering, University of Shanghai for Science and Technology, Shanghai 200093, P.R. China \\ $\square$ Corresponding author: Dr. Ting Liu, The Affiliated Hospital of Qingdao University, No. 16, Jiangsu Road, Southern Distinct, Qingdao 266000, Shandong \\ Province, P.R. China. E-mail: liuting27@126.com; Tel: +86-17717044492
}

(C) The author(s). This is an open access article distributed under the terms of the Creative Commons Attribution License (https://creativecommons.org/licenses/by/4.0/). See http://ivyspring.com/terms for full terms and conditions.

Received: 2020.06.01; Accepted: 2021.03.22; Published: 2021.07.13

\begin{abstract}
Patients suffering from breast cancer $(\mathrm{BC})$ still have a poor response to treatments, even though early detection and improved therapy have contributed to a reduced mortality. Recent studies have been inspired on the association between microRNAs ( $\mathrm{miRs}$ ) and therapies of BC. The current study set out to investigate the role of miR-216b in BC, and further analyze the underlining mechanism. Firstly, hexokinase 2 (HK2) and miR-216b were characterized in BC tissues and cells by RT-qPCR and Western blot assay. In addition, the interaction between HK2 and miR-216b was analyzed using dual luciferase reporter assay. $B C$ cells were further transfected with a series of miR-216b mimic or inhibitor, or siRNA targeting HK2, so as to analyze the regulatory mechanism of miR-216b, HK2 and mammalian target of rapamycin (mTOR) signaling pathway, and to further explore their regulation in BC cellular behaviors. The results demonstrated that HK2 was highly expressed and miR-216b was poorly expressed in BC cells and tissues. HK2 was also verified as a target of miR-216b with online databases and dual luciferase reporter assay. Functionally, miR-216b was found to be closely associated with BC progression via inactivating mTOR signaling pathway by targeting HK2. Moreover, cell viability, migration and invasion were reduced as a result of miR-216b upregulation or HK2 silencing, while autophagy, cell cycle arrest and apoptosis were induced. Taken together, our findings indicated that miR-216b down-regulates HK2 to inactivate the mTOR signaling pathway, thus inhibiting the progression of $B C$. Hence, this study highlighted a novel target for BC treatment.
\end{abstract}

Key words: MicroRNA-216b; HK2; mTOR signaling pathway; Breast cancer; Autophagy

\section{Introduction}

Breast cancer $(\mathrm{BC})$ is a heterogeneous disease of varying subtypes, and each subtype presents with a different overall survival $[1,2]$. Over the years, age, height, reproductive factors (e.g., nulliparity, older age at first birth), use of exogenous hormones, and family history have been elucidated as risk factors for BC. In addition, lifestyle factors such as alcohol consumption, lack of physical activity, and postmenopausal obesity have also been attributed to occurrence of BC to some degree [3]. Hence, early detection is tantamount in controlling the disease mortality all over the world [4]. Accurate estimation for the prognosis of each case would both offer beneficial clinical decision-making and individualized design of follow-up management after surgical treatment [5]. Moreover, some patients still exhibit poor responses to treatment, even though early detection and improved therapy have contributed to reduced mortality [6]. Fortunately, the role of microRNAs (miRs) on the development of BC has been investigated in recent years [7], and has been highlighted to offer promising therapeutic targets for patients plagued by BC.

One such miR, miR-216b is known to be 
extensively implicated in a multitude of cellular processes, including proliferation, invasion, and epithelial-mesenchymal-transition (EMT) [8]; and miR-216b has also been identified to function as a tumor suppressor in various human cancers $[9,10]$. In addition, miR-216b also confers a tumor suppressive role in BC [11]. A prior study has also shown that miR-216b can inhibit the proliferation of cervical cancer cells by targeting FOXM1, and miR-216b can further down-regulate FOXM1 downstream targets or its key regulators $\mathrm{pRb}, \mathrm{c}-\mathrm{myc}$ and cyclinD1 [12]. On the other hand, the hexokinase 2 gene (HK2) has also been associated with cell migration in BC [13]. HK2, a regulator of glucose metabolism, is essential for tumor initiation and maintenance [14]. More notably, miR-216b is known to directly-target the 3'-UTR of HK2 to suppress uveal melanoma tumor growth [15]. However, the relationship between miR-216b and HK2 in BC remains to be defined.

The mammalian target of rapamycin (mTOR) signaling pathway is widely-involved in cellular processes, such as cell growth, proliferation, cellular metabolism, protein synthesis, gene transcription, as well as cell death including apoptosis, autophagy, and necroptosis [16]. Moreover, published literature has also shown that the Akt/mTOR signaling pathway contributes to the inhibitory effects of miR-147 on cell proliferation, invasion and migration in BC [17]. Strikingly, miR-216b was previously demonstrated to influence the development of endometrial cancer through the mTOR signaling pathway [18]. These findings provided a potential network of miR-216b, HK2 and mTOR signaling pathway in BC. Hence, the current study set out to test the hypothesis that miR-216b may promote autophagy and apoptosis of $\mathrm{BC}$ cells via regulation of the mTOR signaling pathway and by targeting HK2.

\section{Results}

\section{The potential functional significance of miR-216b and HK2 in BC progression}

Firstly, through the GEPIA2 database, it was revealed that HK2 was highly expressed in BC tissues compared with normal tissues (Fig. 1A). Next, BC-related disease genes were searched and the top 10 genes were selected (Supplementary Table 1). The analysis of PPIs between HK2 and the known disease genes (Fig. 1B; Supplementary Table 2) showed that HK2 exhibited close interactions with disease genes (AKT1 and PTEN). Moreover, HK2 was recently found to be closely related to tumor progression [19]. Amparo et al. reported that HK2 is up-regulated in malignant glioma, and cell proliferation in malignant glioma relies on glycolysis [20]. In addition, the involvement of HK2 has also been documented in esophageal squamous cancer, pancreatic cancer and liver cancer [21-23]. Also, HK2, as a key gene in glycometabolism, has been previously highlighted as a promising therapeutic target for tumors [24, 25]. All above data indicated that HK2 might play a key role in tumor progression. However, mechanism involving the roles HK2 in BC remains unclear. On the other hand, AKT1 and PTEN are well-known key factors of the PI3K-AKT-mTOR signaling pathway, which is closely related to $\mathrm{BC}$ according to the KEGG database (map05224). Moreover, studies have elucidated that cell proliferation, migration and invasion can all be modulated by the PI3K-AKT-mTOR signaling pathway [26-28]. Additionally, activated PI3K-AKT-mTOR signaling pathway has been found to promote epithelial-mesenchymal transition (EMT) and angiogenesis in triple-negative BC [29].

To further explore the upstream regulator of HK2 in BC, we predicted the miRs that could possibly regulate the HK2 gene. Results from the miRWalk database indicated a total of 2089 potential miRs that could regulate HK2. In addition, miR pre-computed tool provided by the Computational Medicine Center (RNA22 tool) predicted another such 2039 miRs. The top 1000 miRs highly-related to HK2 were selected from each database. Additionally, $66 \mathrm{miRs}$ were also predicted from the miRDB Database and $33 \mathrm{miRs}$ from microRNA.org. Intersections of the above prediction results showed that has-miR-216b was found in all 4 databases (Fig. 1C). Besides, studies have illustrated that has-miR-216b inhibits tumor growth in colorectal cancer, non-small cell lung cancer and cervical cancer [12, 30, 31]. Consequently, miR-216b was suggested to probably suppress $B C$ progression via inhibition of $H K 2$ in terms of all above analyses and documentation.

\section{HK2 is highly expressed in BC}

The protein expression patterns of HK2 in BC and adjacent normal tissues were detected using immunohistochemistry assay. The results showed that HK2 was highly expressed in BC tissues with the cytoplasm stained with tan coloration, while HK2 was poorly expressed in adjacent normal tissues (Fig. 2A). The positive rate of HK2 protein was calculated to be $73.91 \%(102 / 138)$ in BC tissues, which was significantly higher than $29.71 \%(41 / 138)$ of the adjacent normal tissues $(p<0.05)$ (Fig. 2B). RT-qPCR and Western blot analysis indicated that the mRNA and protein expression of HK2 was elevated in BC tissues relative to adjacent normal tissues $(p<0.05)$ (Fig. 2C-D). 
A

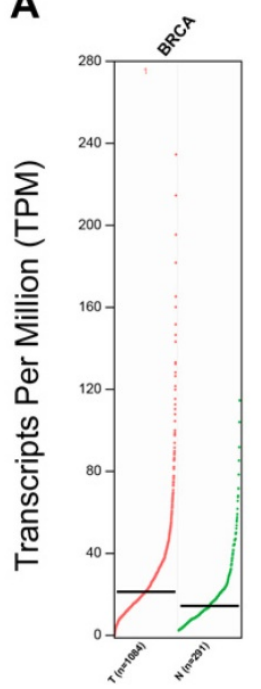

B

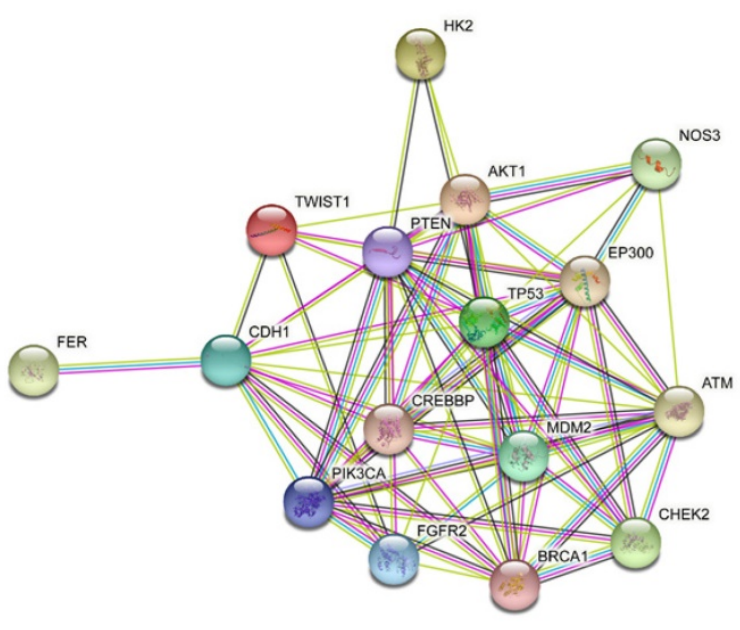

C

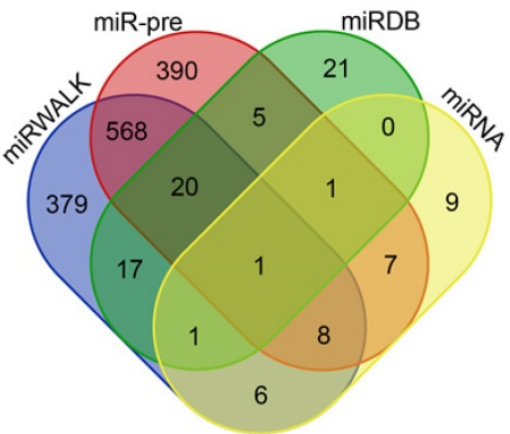

Figure 1. Screening of BC-related genes, signaling pathways and miRs. A, GEPIA2 database reveals expression of $H K 2$ in BC tissues and normal tissues. B, the interactions between HK2 and known disease genes analyzed on STRING database; the thickness of line between two genes means the reliability of interaction between two genes, the thicker line indicates a higher reliability; $\mathrm{C}$, the miRs targeting HK2 predicted by miRWalk and Computational Medicine Center using miR-pre, miRDB Database and microRNA.org.

A

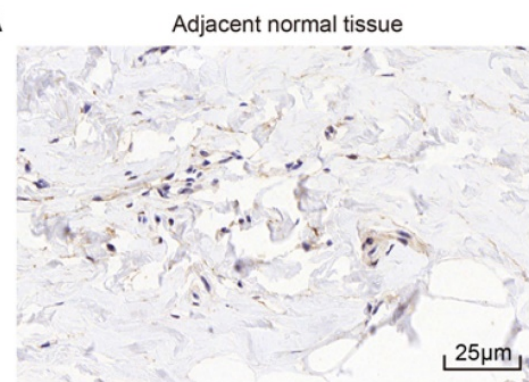

C

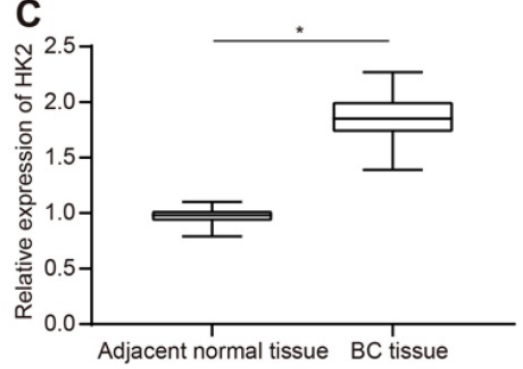

BC tissue

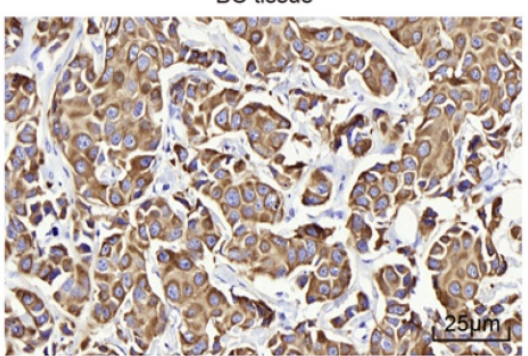

D

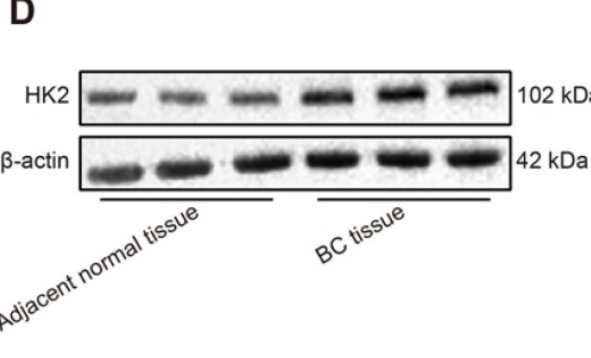

B
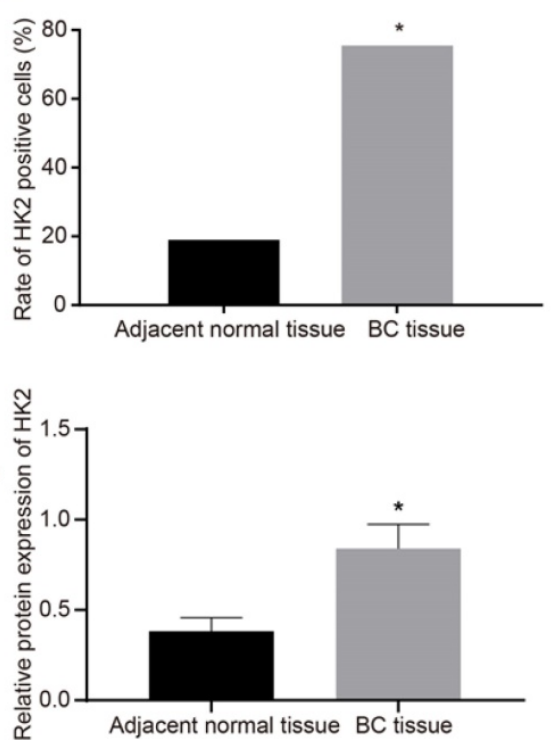

Figure 2. HK2 is highly expressed in BC tissues. A, immunochemical staining for detection of HK2 expression in $B C$ and adjacent normal tissues ( $\times 400$ ); $B$, positive rate of HK2; $n=138$, the data were analyzed using chi-square test. C, HK2 mRNA expression in BC and adjacent normal tissues determined by RT-qPCR; D, HK2 protein expression in BC and adjacent normal tissues measured by Western blot assay and related statistical data. ${ }^{*}, p<0.05$ vs. the adjacent normal tissues.

\section{MiR-216b binds to HK2 3'UTR}

RT-qPCR and RNA-fluorescence in situ hybridization (FISH) results indicated that miR-216b expression was significantly lower in $\mathrm{BC}$ tissues than in the adjacent normal tissues $(p<0.05)$ (Fig. 3A-B), which were negatively-correlated with the expression of HK2 (Fig. 3C). Furthermore, a target prediction database (microRNA.org) further predicted the presence of a specific binding region between HK2 3'UTR and the miR-216b sequence. Subsequent dual luciferase reporter assay results (Fig. 3D) showed that the luciferase activity of $\mathrm{pHK} 2-\mathrm{Wt}$ was significantly inhibited by miR-216b mimic compared with the control group $(p<0.05)$. After transfect with miR-216b mimic or inhibitor, miR-216b and HK2 levels were detected using RT-qPCR and Western blotting. The results showed that compared with mimic-NC, miR-216b mimic significantly inhibited the HK2 expression, while miR-216b inhibition brought about the opposite effects on HK2 expression $(p<0.05)$ (Fig. 3E-F). The aforementioned results indicated that miR-216b could target HK2 and regulate its expression. 
A

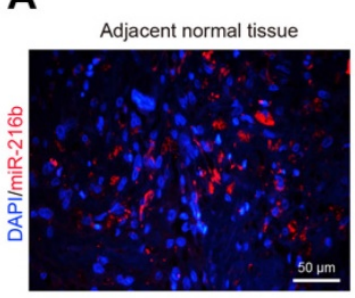

C

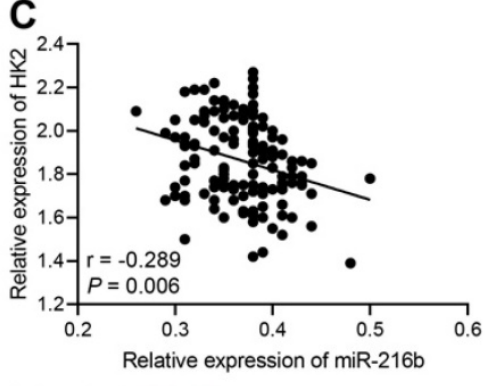

E

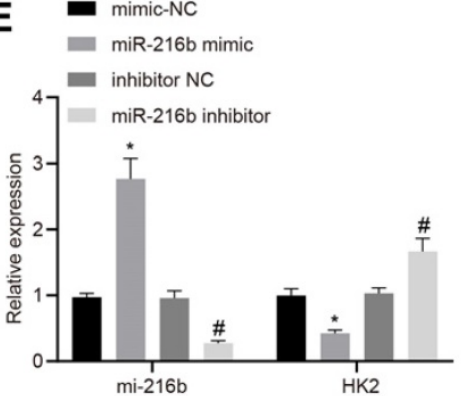

BC tissue

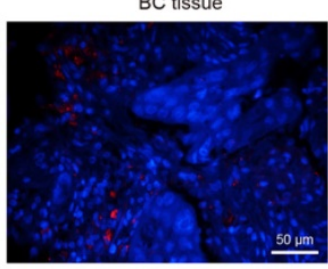

D

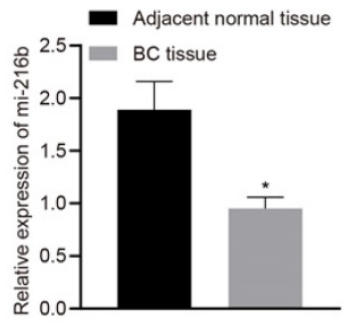

B

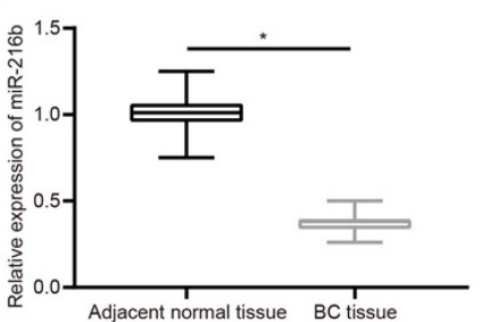

3' uggcuccACGGGCGUCUCUAA-5 MUT

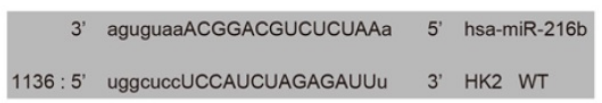

$\mathbf{F}$

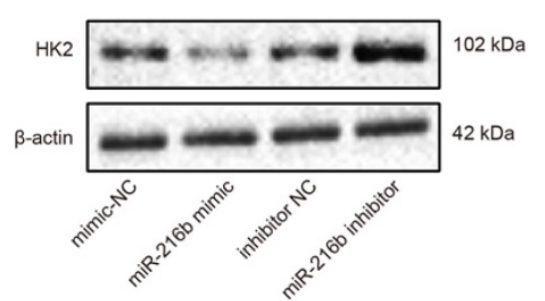

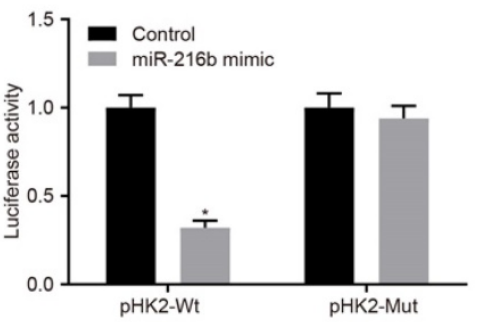

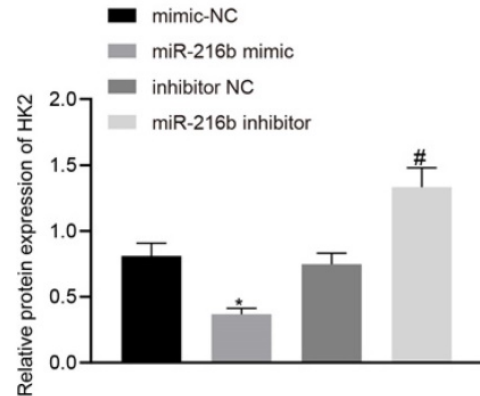

Figure 3. HK2 is identified as a target gene of miR-216b. A, miR-216b expression determined by FISH $(\times 200)$; B, miR-216b expression in BC and adjacent normal tissues determined by RT-qPCR; C, correlation analysis of miR-216b and HK2 expression in BC tissues. D, the binding site of miR-216b to the HK2 3 'UTR from the target prediction program microRNA (left); the luciferase activity after treatment by a combination of miR-216b mimics and HK2-3'UTR-wt (right); E, miR-216b and HK2 expression after miR-216b expression was altered detected by RT-qPCR; F, HK2 expression after miR-216b expression was altered detected by Western blotting; the experiment was repeated for three times. $*, p<0.05$ vs. vs. Normal tissues, the control group, mimic-NC or inhibitor NC groups.

\section{Low expression of miR-216b and high expression of $\mathrm{HK} 2$ are associated with BC progression}

As shown in Supplementary Table 3, miR-216b expression and HK2 mRNA expression exhibited no association with age and histological type. Meanwhile, the miR-216b expression was found to be significantly lower and HK2 expression was higher in patients with a lesion size $>2 \mathrm{~cm}$, lymph node metastasis (LNM) and at III stage than in those with lesion size $\leq 2 \mathrm{~cm}$, without LNM and at I/II stage ( $p<$ $0.05)$. These findings illustrated that low miR-216b expression and high HK2 expression was associated with BC progression. RT-qPCR and Western blot analysis were then performed to determine the expression patterns of apoptotic factors (Bax and Bcl-2), autophagy-related genes (Beclin1, LC3 and MMP-9), and mTOR signaling pathway-related genes (mTOR and 4EBP1). The results showed that mRNA and protein expression of Beclin1, Bax and LC3 was significantly lower, while that of mTOR, Bcl-2, 4EBP1 and MMP-9 was higher in BC tissues compared to that in adjacent normal tissues $(p<0.05)$ (Fig. 4A, B), suggesting decreased cell apoptosis and autophagy and activated mTOR signaling pathway in BC tissues.

\section{MiR-216b down-regulates HK2 to block the mTOR signaling pathway}

For cell line selection, miR-216b expression patterns were determined in BC cell lines (MCF-7, MDA-MB-468) and normal human mammary epithelial cell line (MCF-10A) using RT-qPCR. The results showed that, compared with that of MCF-10A cell line, miR-216b expression was decreased in MCF-7 cell line, and further reduced in the MDA-MB-468 cell line $(p<0.05)$ (Fig. 5A); therefore, MDA-MB-468 was used for subsequent experimentation.

As demonstrated by the results from Western blot analysis, siHK2-1 was selected for HK2 knock-down experiments due to more remarkable reduction induced by transfection with siHK2-1 (Fig. 5B). There were no significant differences in the expression of mRNA and protein of miR-216b and HK2, mTOR and 4EBP1 between the blank group and 
NC groups, with no significant differences in the expression of p-mTOR and p4EBP1 $(p>0.05)$. Compared with the NC group, the expression of $\mathrm{miR}-216 \mathrm{~b}$ in the miR-216b mimic group was found to be increased significantly $(p<0.05)$, while that of HK2, mTOR and 4EBP1 was decreased, and the expression of p-mTOR and p4EBP1 was also reduced $(p<0.05)$. Meanwhile, the expression of miR-216b in the miR-216b inhibitor group was decreased $(p<0.05)$, while HK2, mTOR and 4EBP1 were up-regulated, and the expression of p-mTOR and p4EBP1 was elevated $(p<0.05)$. Moreover, HK2, mTOR and 4EBP1 expression in si-HK2 group was decreased, and p-mTOR and p4EBP1 expression was also reduced significantly $(p<0.05)$, while that of miR-216b was not significantly altered $(p>0.05)$. After treatment with si-HK2 + miR-216b inhibitor, the expression of miR-216b and HK2 was decreased $(p<0.05)$, while that of mTOR, 4EBP1 and p-mTOR, p4EBP1 did not change significantly $(p>0.05)$ (Fig. 5C-D).

Previous results showed that HK2 was highly expressed in cancer tissues, and the expression of p-mTOR was decreased in BC cells when HK2 was silenced. As a result, we hypothesized that HK2 was positively correlated with $\mathrm{p}-\mathrm{mTOR}$, and then detected the expression patterns of $\mathrm{p}$-mTOR in $\mathrm{BC}$ tissues and adjacent normal tissues using immunohistochemistry. The results showed that p-mTOR was highly expressed in cancer tissues and positively correlated with HK2 (Fig. 5E-F).

To validate the involvement of the miR-216b-HK2-mTOR regulatory axis in breast cancer cells, we performed a rescue experiment. In MCF-7 cells, RT-qPCR was performed to detect the expression patterns of miR-216b and HK2, and Western blot was applied to detect the expression patterns of mTOR, p-mTOR, 4EBP1 and p4EBP1 in HK2 and mTOR pathways. The results showed that compared with mimic-NC + oe-NC treatment, miR-216b was upregulated but the expression of HK2, p-mTOR and p4EBP1 was inhibited in the miR-216b mimic + oe-NC group Inhibition of HK2, p-mTOR and p4EBP1 expression by miR-216b mimic + oe-NC could be restored by miR-216b mimic + oe-HK2 $(p<$ 0.05) (Fig. 5G-H).

\section{MiR-216b upregulates Beclin 1, Bax and LC3 but down-regulates $\mathrm{Bcl}-2$ and MMP-9}

To further investigate whether miR-216b could affect $\mathrm{BC}$ progression, the cellular expression patterns of Beclin1, Bax, Bcl-2, LC3 and MMP-9 were determined following activation or depletion of miR-216b, or silencing of HK2. As depicted in Fig. 6A, $\mathrm{B}$, the mRNA expression of Beclin1, Bax and LC3, protein expression of Beclin1 and Bax and ratio of LC3 II/I were significantly elevated by miR-216b mimic or siHK2 transfection, while that of Bcl-2 and MMP-9 was markedly down-regulated $(p<0.05)$; and these trends could be reversed following treatment with miR-216b inhibitor $(p<0.05)$. Meanwhile, the co-transfection of siHK2 and miR-216b inhibitor brought about no significant changes in relation to the aforementioned expression levels $(p>0.05)$. These findings indicated that miR-216b upregulated Beclin1, Bax and LC3 and downregulated Bcl-2 and MMP-9.
A

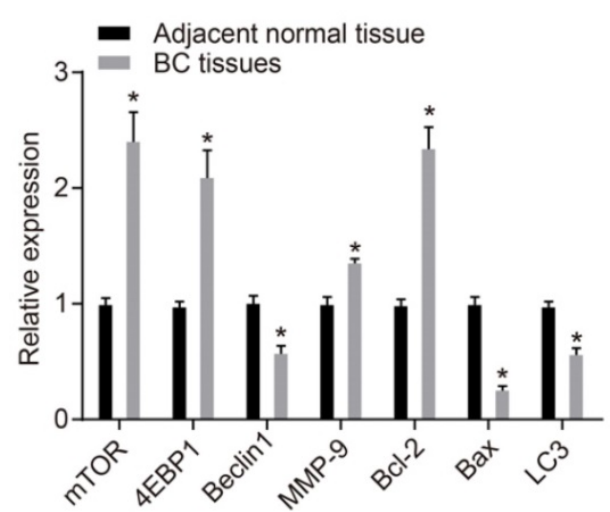

B

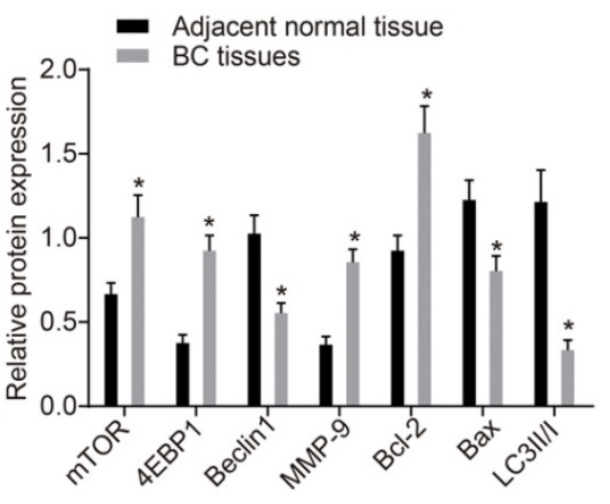

Figure 4. $m$ TOR signaling pathway is activated in BC. A, mRNA expression of mTOR, Bax, Bcl-2, LC3, Beclin 1, 4EBP1 and MMP-9, as determined by RT-qPCR; B, Western blot analysis of the protein expression of mTOR, Bax, Bcl-2, LC3, Beclin l, 4EBPI and MMP-9; $\mathrm{n}=138$, the data were analyzed using paired $t$ test. *, $p<0.05$ compared with adjacent normal tissues. 
A
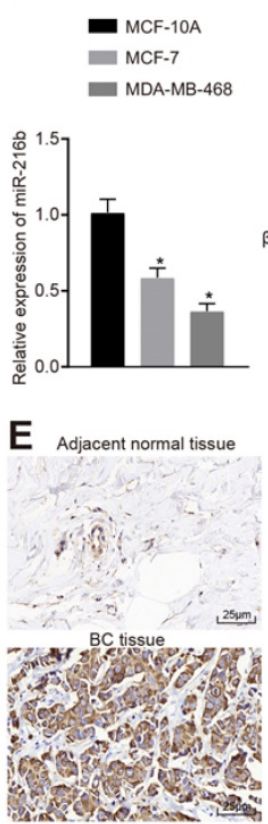

B

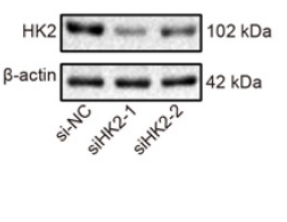

Adjacent normal tissue

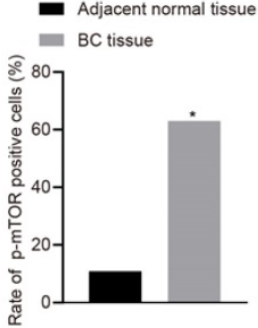

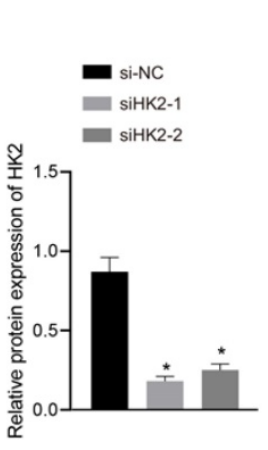

$\mathbf{F}$

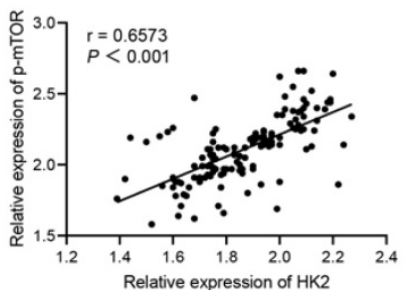

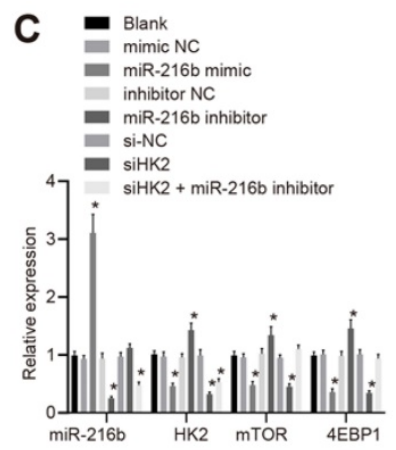

G

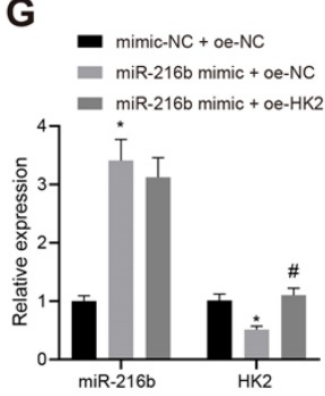

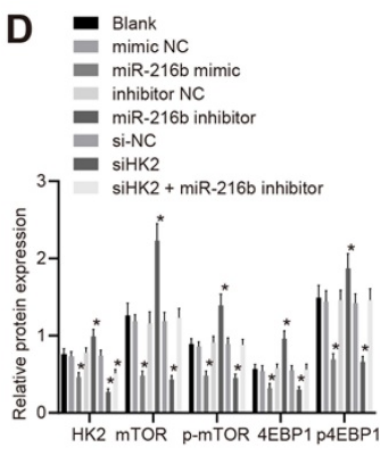

H

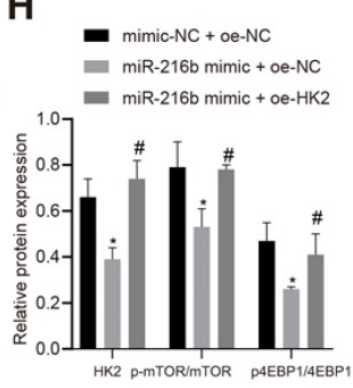

Figure 5. MiR-216b targets HK2 to block the mTOR signaling pathway. A, miR-216b expression among three tested BC cell lines; $\mathrm{B}$, silencing efficiency of siHK2-1 and siHK2-2. C, RT-qPCR assay for determination of miR-216b and mRNA expression of HK2, mTOR and 4EBP1; $D$, the protein expression of HK2, mTOR and 4EBP1, along with the extent of $m$ TOR and 4EBP1 phosphorylation measured by Western blot analysis; E, p-mTOR expression in tissues detected by IHC ( $\times 400)$; F, correlation analysis of $\mathrm{p}$-mTOR and HK2; $\mathrm{G}$, miR-216b and HK2 expression detected by RT-qPCR; H, the protein expression of HK2, mTOR and 4EBP1, along with the extent of mTOR and 4EBP1 phosphorylation measured by Western blot analysis; the experiment was repeated for three times; data were analyzed using one-way ANOVA with Tukey's post hoc test. *, $p<0.05$ compared with the cells transfected with mimic NC + oe-NC; \#, $p<0.05$ compared with miR-216b mimic +oe-NC.
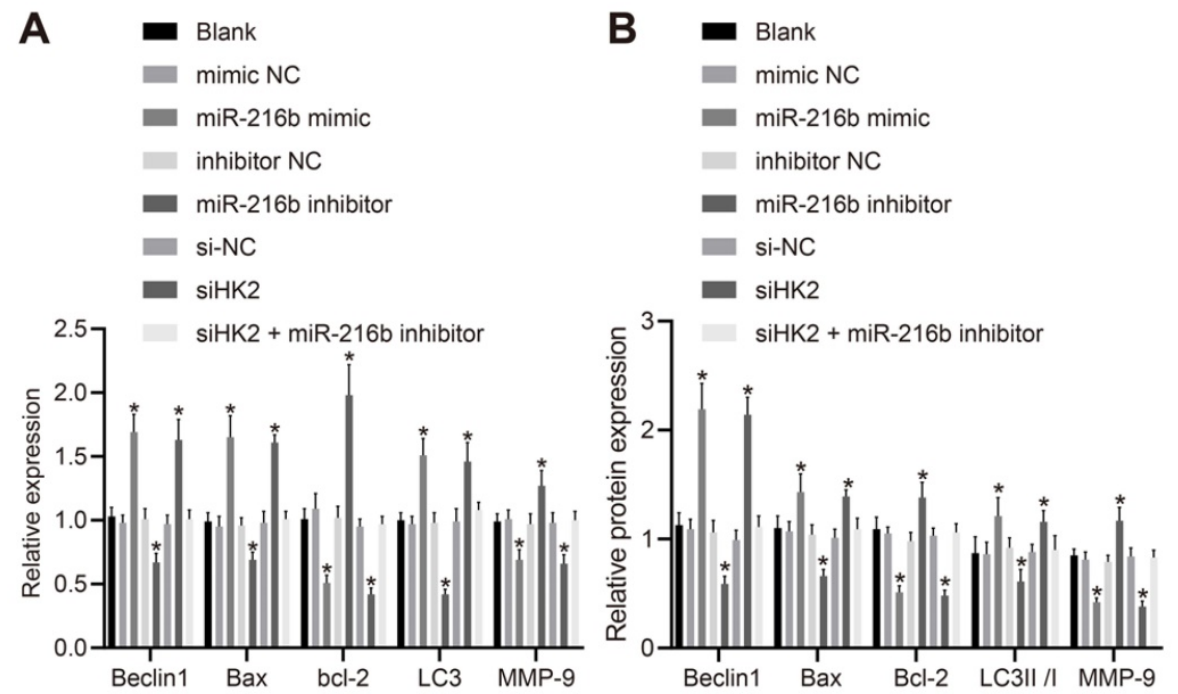

Figure 6. MiR-216b regulates expression of Beclin 1, Bax, Bcl-2, MMP-9, LC3, LC3 I and LC3 II. A, mRNA expression of Beclin 1, LC3, Bax, Bcl-2 and MMP-9 determined by RT-qPCR; B, the protein expression of Beclin I, Bax, Bcl-2, LC3 I, LC3 II and MMP-9 measured by Western blot analysis; the experiment was repeated for three times; data were analyzed using one-way ANOVA. *, $p<0.05$ compared with the cells transfected with mimic NC, inhibitor NC or siNC.

\section{MiR-216b inhibits BC cell proliferation, migration, invasion by targeting HK2}

Next, CCK-8 assay was applied to detect the cell proliferation at the $0 \mathrm{~h}, 24^{\text {th }} \mathrm{h}, 48^{\text {th }} \mathrm{h}$ and $72^{\text {nd }} \mathrm{h}$ time points after transfection. Compared with mimic-NC + oe-NC, cell proliferation was found to be inhibited in the miR-216b mimic + oe-NC group. Meanwhile, miR-216b mimic + oe-HK2 could restore the inhibition of cell proliferation by miR-216b mimic + oe-NC $(p<$
0.05) (Fig. 7A). In addition, scratch assay and Transwell assay were performed to assess the possible regulatory effects of miR-216b and $\mathrm{HK} 2$ on cell migration and invasive abilities of $\mathrm{BC}$. The cell migration (Fig. 7B) and invasion (Fig. 7C) were found to be inhibited in the presence of miR-216b mimic (both $p<0.05$ ), which was negated by overexpression of HK2. These findings demonstrated that miR-216b inhibited the cell proliferation, migration and invasiveness in BC by down-regulating HK2 in BC. 


\section{MiR-216b promotes BC cell cycle arrest and apoptosis by targeting HK2}

Furthermore, the effect of miR-216b and HK2 on cell cycle distribution and apoptosis of BC cells was evaluated using flow cytometry analyses of PI single staining and Annexin V-FITC/PI double staining. Compared with mimic-NC + oe-NC, treatment with miR-216b mimic + oe-NC significantly blocked the cells at the G1 phase. Meanwhile, miR-216b mimic + oe-HK2 could alleviate the arrest of cells at the G1 phase induced by miR-216b mimic + oe-NC $(p<0.05)$ (Fig. 8A). The results of apoptosis experiment showed that compared with mimic-NC + oe-NC, apoptosis in the miR-216b mimic + oe-NC group was increased; the apoptosis of cells after miR-216b mimic + oe-HK2 treatment could be alleviated by miR-216b mimic + oe-NC $(p<0.05)$ (Fig. 8B). These findings demonstrated that upregulation of miR-216b promoted cell cycle arrest as well as cell apoptosis in BC by targeting HK2.

\section{MiR-216b promotes BC cellular autophagy by targeting $\mathrm{HK2}$}

In addition, the effects of miR-216b and HK2 on autophagy of BC cells MCF-7 and MDA-MB-468 cells were analyzed using MDC assay. As demonstrated in Fig. 9A, B, compared with mimic-NC + oe-NC, autophagy was found to be increased in the miR-216b mimic + oe-NC group. The autophagy of cells in the
miR-216b mimic + oe-HK2 group could be alleviated by miR-216b mimic + oe-NC $(p<0.05)$. These findings demonstrated that upregulation of miR-216b promotes cell autophagy in BC by targeting HK2.

\section{MiR-216b inhibits tumor growth in vivo}

Lastly, MDA-MB-468 cells transfected with miR-216b mimic or mimic NC were subcutaneously injected into nude mice so as to explore the effect of miR-216b and HK2 on in vivo oncogenicity of BC cells. The mice were euthanized once the tumors were formed. It was observed that the tumors in the miR-216b mimic group were smaller than those in the NC group (Fig. 10A). Volume and mass of the tumors were also measured, and the results were shown in Fig. 10B-C. The volume and mass of tumors in the miR-216b mimic group were found to be reduced, which suggested that miR-216b inhibited BC progression in vivo. The expression of related genes in each group of mice was further detected using RT-qPCR and Western blot (Fig. 10D, E). Compared with the mimic-NC group, the expression of HK2, mTOR, 4EBP1, bcl-2, MMP-9, p-mTOR, and p-4EBP1 was noted to be decreased in the miR-216b mimic group $(p<0.05)$, while that of Beclin1, Bax, LC3 and LC3II/I was increased $(p<0.05)$. Collectively, these findings indicated that upregulation of miR-216b restrained in vivo tumor growth of $\mathrm{BC}$.
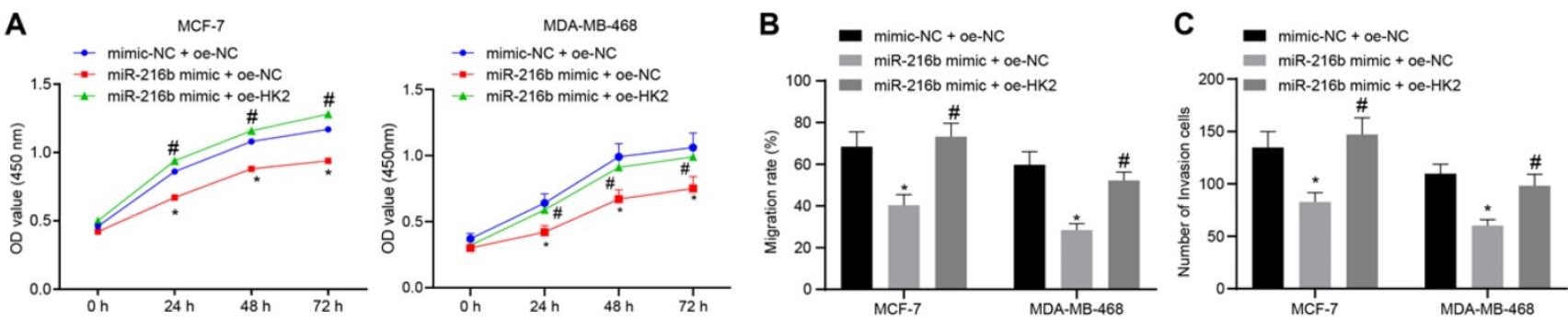

Figure 7. miR-216b inhibits $\mathrm{BC}$ cell proliferation, migration and invasion by targeting HK2. A, BC cell proliferation in response to miR-216b upregulation or HK2 overexpression assessed by CCK-8; B, BC cell migration detected by scratch test; $C, B C$ cell invasion detected by Transwell assay; the experiment was repeated for three times; data were analyzed using one-way ANOVA. *, $p<0.05$ compared with the cells transfected with mimic NC + oe-NC; \#, $p<0.05$ compared with miR-216b mimic +oe-NC.
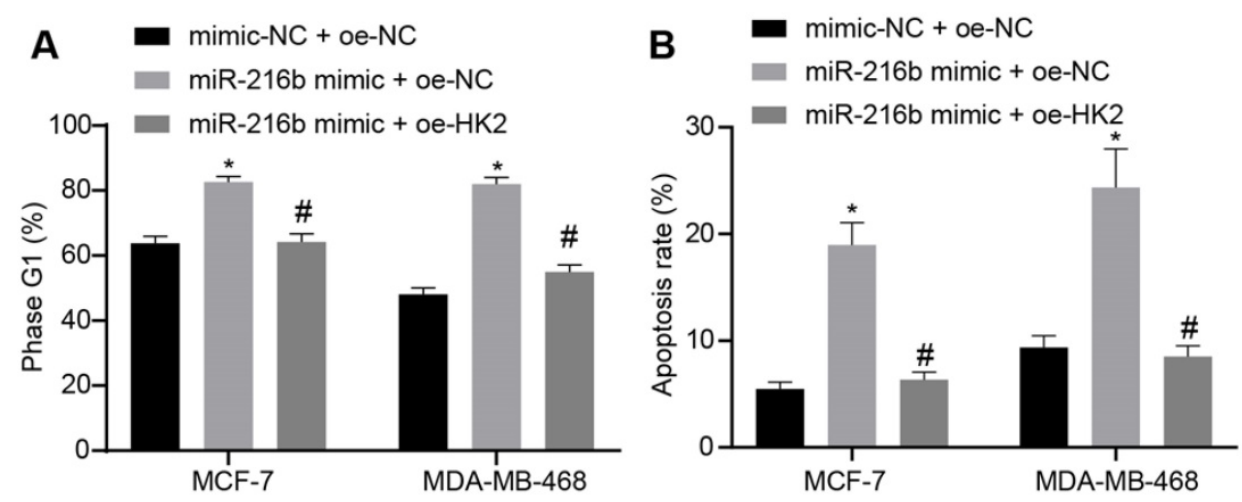

Figure 8. miR-216b promotes cell cycle arrest and apoptosis by targeting HK2. A, flow cytometric analysis of cell cycle distribution; B, apoptosis rate after miR-216b upregulation and/or HK2 overexpression; the experiment was repeated for three times; data were analyzed using one-way ANOVA. *, $p<0.05$ compared with the cells transfected with mimic $\mathrm{NC}+$ oe-NC; \#, $p<0.05$ compared with miR-216b mimic +oe-NC. 

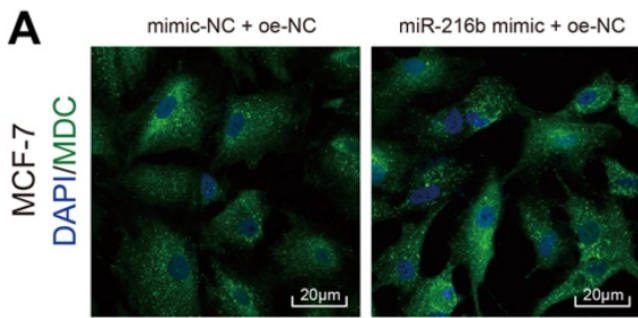

miR-216b mimic + oe-HK2

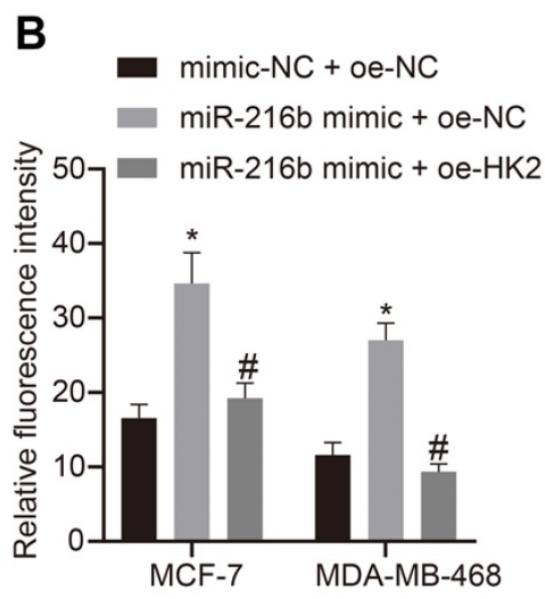

Figure 9. miR-216b promotes cell autophagy by targeting HK2. A, representative images of cell autophagy shown by MDC staining $(\times 500)$ images; $B$, statistical data. The images of DAPI staining indicates the nuclei, and merge filed indicates the presence of MDC-stained autolysosomes and DAPI-stained nuclei. B, the intensity of formed autolysosome.*, $p<0.05$ compared with the cells transfected with mimic NC + oe-NC; \#, $p<0.05$ compared with miR-216b mimic +oe-NC.
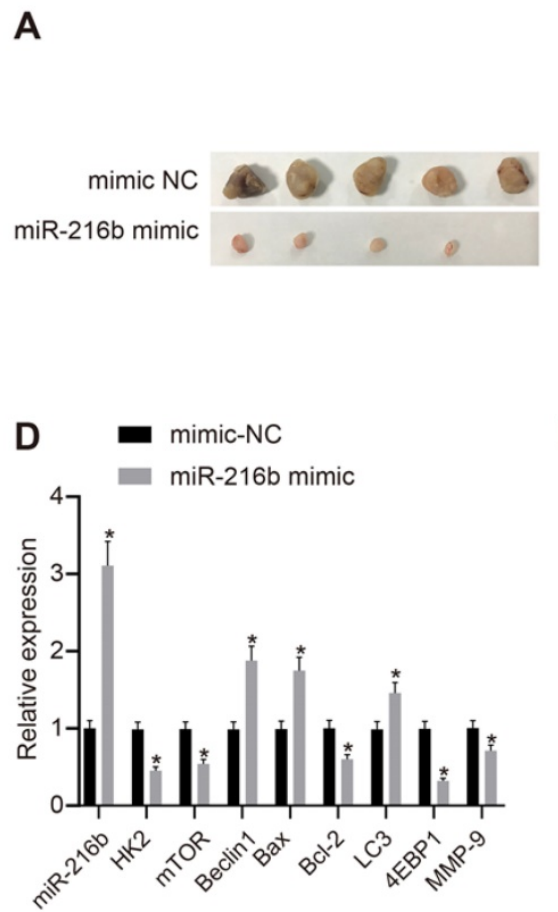

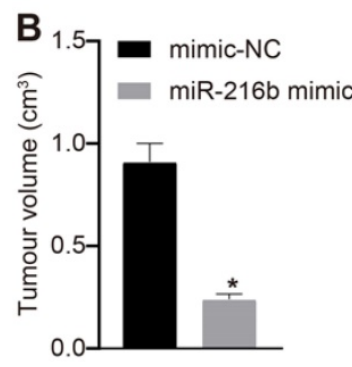

E

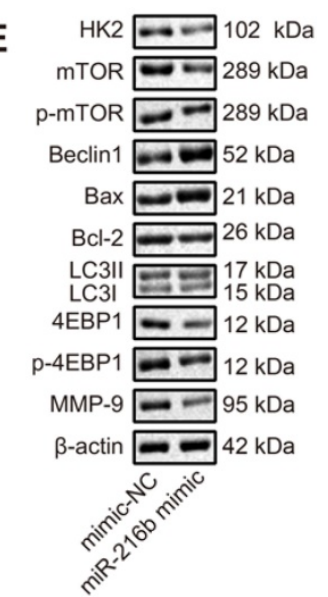

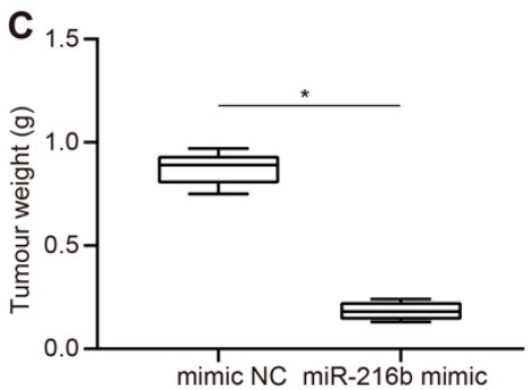

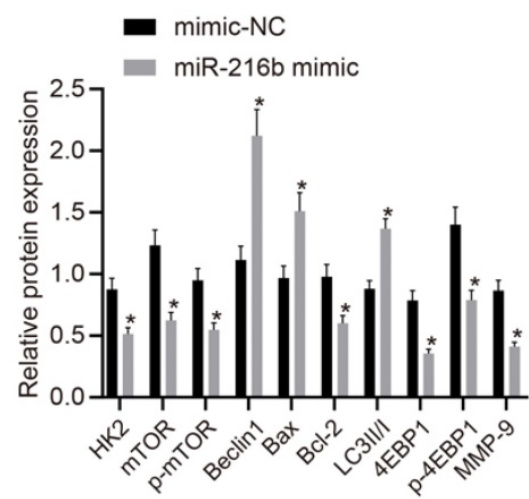

Figure 10. miR-216b inhibits the tumor growth in nude mice. $A$, representative images of tumors in nude mice after miR-216b upregulation; $n=5$ in the mimic-NC group; $n=$ 4 in the miR-216b mimic group; B, tumor volume in nude mice after miR-216b upregulation; $C$, tumor weight in nude mice after miR-216b upregulation; $D$, miR-216b expression and mRNA expression of HK2, mTOR, Beclin 1, LC3, Bax, Bcl-2, 4EBP1 and MMP-9 after miR-216b upregulation determined by RT-qPCR; E, protein expression of HK2, mTOR, p-mTOR, Beclin 1, Bax, Bcl-2, LC3 I, LC3 II, 4EBP1, P-4EBPI and MMP-9 measured by Western blot analysis; the data were analyzed using unpaired $t$ test. *, $p<0.05$ compared with the nude mice injected with mimic-NC-transfected cells.

\section{Discussion}

$\mathrm{BC}$ is one of the leading causes of cancer-related death among women worldwide [32]. Although 5 -year disease-specific survival rates have been improved over the last few decades, a considerable proportion of BC cases are still detected at advanced stages, and show a higher mortality rate due to the advent of drug resistance and increased risk of tumor recurrence [33]. Therefore, it is quite necessary to find more effective treatment regimens for BC patients. In lieu of this, the current study set out to uncover the effects of miR-216b on the cellular behavior of BC cells, and obtained findings revealed that miR-216b suppressed BC invasion and migration, and further induced autophagy and apoptosis via the mTOR signaling pathway by targeting HK2. 


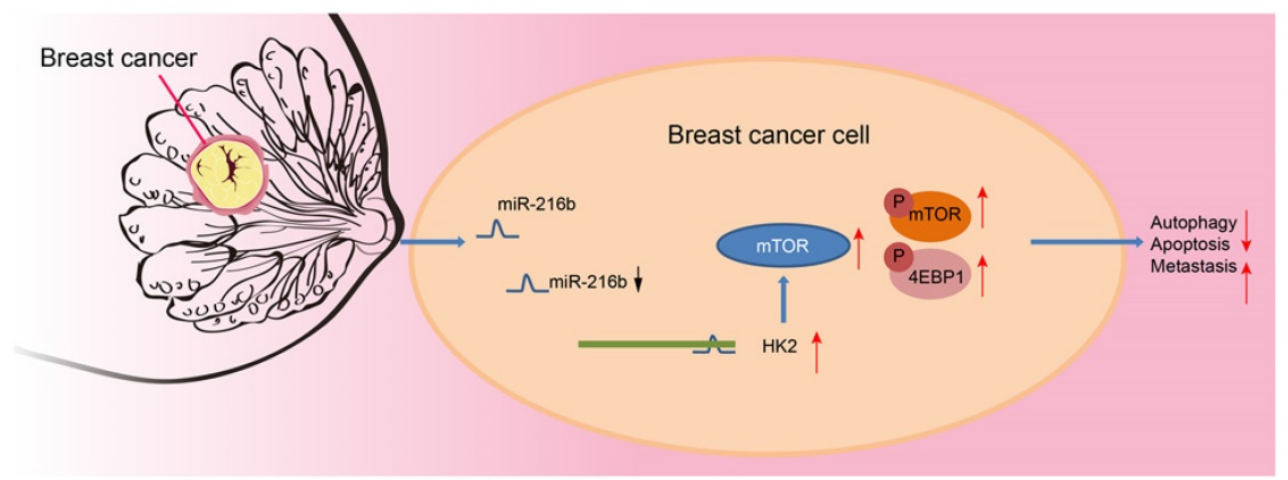

Figure 11. Mechanism. miR-216 down-regulates HK2 to inactivate the mTOR signaling pathway, thus inhibiting the progression of BC.

Firstly, our findings demonstrated that miR-216b was poorly-expressed in BC. Similarly, Deng et al. documented down-regulated expression of miR-216 in nasopharyngeal carcinoma, which were inversely-correlated with tumor metastasis and clinical stage [34]. Moreover, the current study unraveled that miR-216b was associated with the progression of $\mathrm{BC}$, as attributed to suppressed $\mathrm{BC}$ invasion and migration abilities and induction of autophagy and apoptosis upon over-expression of miR-216b. Meanwhile, a previous study also suggested that lung cancer cells diminished the miR-216b levels to induce the production of autophagy protein Beclin-1 to augment cell survival, which is in accordance with our findings [35]. Strikingly, in contrast to other protein kinase RNA-like ER kinase-regulated miRs, miR-216b enhances unfolded protein response-associated apoptosis through regulating a newly identified target, c-Jun [36]. These findings and evidence together suggest that miR-216b attenuates the progression of $\mathrm{BC}$.

Interestingly, several studies have highlighted the association of HK2 with cell proliferation and/or progression in BC [37]. Strikingly, immunochemical staining results in our study illustrated that HK2 was highly expressed in BC tissues. Moreover, we further identified that HK2 expression was associated with clinical stage, LNM and tumor diameter in BC. As a key glycolytic enzyme for the Warburg effect, HK2 is known to be upregulated in numerous malignancies, and to catalyze the irreversible first step of glucose metabolism [38]. In addition, another study has also documented the enrichment of HK2 in cancer cells, wherein this enrichment resulted in high glycolytic rates in tumors [39]. HK2 is also demonstrated in the metastatic process of pancreatic ductal adenocarcinoma (PDAC) [40]. More importantly, findings in our study further demonstrated that miR-216b targets HK2 and negatively modulates its expression to prevent the progression of $\mathrm{BC}$.
Moreover, further experimentation indicated that miR-216b negatively modulate HK2 expression to suppress cell viability, invasion and migration, as well as to induce autophagy and apoptosis. On the other hand, activation of mTOR signaling pathway has also been previously implicated in astragaloside II (AS II)-induced wound healing [41]. Meanwhile, a study has shown that inhibition of the PI3K/Akt/mTOR signaling pathway can positively enhance autophagy in multiple myeloma [42]. As a downstream factor of the mTOR signaling pathway, 4EBP1 is known as an indicator of breast tumorigenesis, interplaying with hormone receptor signaling [43]. In our study, we observed that miR-216b down-regulates mTOR and $4 \mathrm{EBP} 1$, which is indicative of inactivation of the mTOR signaling pathway, which provides evidence for the regulatory role of miR-216b in BC progression.

Altogether, our results provide strong evidence that upregulation of miR-216b enhances autophagy and apoptosis, as well as suppresses invasion and migration of BC cells via blocking the mTOR signaling pathway by targeting HK2. Additionally, in vivo experimentation in our study demonstrated that transplanted neoplasms were inhibited by miR-216b, which highlights its anti-tumor role (Fig. 11). However, further evidence of the detailed mechanisms of how miR-216b functions with HK2 and mTOR is warranted to effectively use our findings to improve the life of patients plagued by BC. Nevertheless, we indicate miR-216b as a promising therapeutic target for $\mathrm{BC}$ by inhibition of HK2 and inactivation of the mTOR signaling pathway.

\section{Patients and Methods}

\section{Ethic statements}

All experiments involving humans were performed with the approval of the Ethics Committee of Xinhua Hospital, and signed informed consents were obtained from all participants before the study. All experiments involving animals were conducted in compliance with the Animal Ethics Committee of 
Xinhua Hospital, and extensive efforts were made to minimize the suffering of the included animals.

\section{Microarray analysis}

Gene expression Profiling Interactive Analysis 2 (GEPIA2) (http://gepia2.cancer-pku.cn) is an updated and enhanced version of GEPIA that supports 198,619 isoforms and 84 cancer subtypes [44]. The expression of HK2 in BC was determined in GEPIA2. Fragments per kilobase million (FPKM) values were transformed into transcripts per kilobase million (TPM) values, which are more comparable between samples. Genotype-tissue expression (GTEx; http://commonfund.nih.gov/GTEx/) provides publicly available gene expression data from 54 normal tissue sites across nearly 1,000 people by RNA sequencing. Normal samples from both TCGA and GTEx (http://commonfund.nih.gov/GTEx/) databases were used for comparisons between cancer and normal tissues.

\section{Protein-protein interaction (PPI) analysis}

Disease genes were retrieved from the DisGeNET database (http://www.disgenet.org/ web/DisGeNET/menu) [45, 46]. Data of BC were retrieved using the key word "breast carcinoma" in the DisGeNET and the top 10 genes were used for subsequent analysis. PPI analysis was performed using the STRING database (https:/ / string-db.org/).

\section{Enrichment analysis of Kyoto Encyclopedia of Genes and Genomes (KEGG) pathways}

Information including gene function and metabolic pathways was obtained from the KEGG database (http://www.kegg.jp/kegg/). Metabolic pathways related to $\mathrm{BC}$ were retrieved using the key word "breast cancer".

\section{MiR prediction}

miRs possessing the ability to regulate this gene were predicted and obtained from the miRWalk database (http://129.206.7.150/), miRDB database (http://www.mirdb.org/mirdb/policy.html), miR pre-computed target prediction using the RNA22 tool (https://cm.jefferson.edu/rna22/Precomputed/Opti onController?species $=$ HomoSapiens\&type $=$ mRNA) and microRNA.org database (http://34.236.212.39/ microrna/home.do). Subsequently, intersections of predicted miR lists were analyzed with the help of a Venn diagram website (http://bioinformatics .psb.ugent.be/webtools/Venn/).

\section{Study subjects}

BC and adjacent normal tissues were collected from 138 female patients (histopathologically confirmed as BC) at the Xinhua Hospital, School of
Medicine, Shanghai Jiao Tong University from November 2016 to September 2018. Patients who received any medicine therapy, chemoradiotherapy or/and immuno-biotherapy before the study were excluded [47]. In addition, BC cell lines (MCF-7 and MDA-MB-468), and normal human mammary epithelial cell line (MCF-10A) were purchased from Biobw (Beijing, China). The MCF-7 cells were maintained in minimum essential medium (MEM) supplemented with $10 \%$ fetal bovine serum (FBS, $10 \mu \mathrm{g} / \mathrm{mL}$ bovine Insulin and $1 \%$ penicillinstreptomycin). MDA-MB-468 cells were cultured in L-15 medium supplemented with 10\% FBS. Meanwhile, the MCF-10A cell line was cultured in DMEM-F12 supplemented with 5\% horse serum, $20 \mathrm{ng} / \mathrm{mL}$ epidermal growth factor (EGF), $100 \mathrm{ng} / \mathrm{mL}$ cholera toxin, $10 \mu \mathrm{g} / \mathrm{mL}$ cells were insulin, and $0.5 \mu \mathrm{g} / \mathrm{mL}$ hydrocortisone. All cells were cultured in a $5 \% \mathrm{CO}_{2}$ incubator at $37^{\circ} \mathrm{C}$.

\section{Immunohistochemistry}

Immunohistochemical staining was performed using previously published methods [48]. Briefly, BC and adjacent normal tissues were probed with the mouse anti-human monoclonal antibody to HK2 (dilution ratio of 1: 200, ab227198, Abcam, Cambridge, MA) and phosphorylated mTOR (p-mTOR; dilution ratio of $1: 100$, ab109268, Abcam) overnight at $4^{\circ} \mathrm{C}$ or at $37^{\circ} \mathrm{C}$ for another $1 \mathrm{~h}$. Meanwhile, biotinylated goat anti-rabbit IgG (ab205718, Abcam) was used as the secondary antibody. Five fields were randomly selected and observed under an optical microscope (Nikon). Judgment criteria for positive-expression were as follows: cell presenting with brown and yellow cytoplasm were regarded to be positively expressed. Five representative high-power fields (positive optical microscope, NIKON, Japan) were also selected for observation and cell counting.

\section{FISH detection}

The paraffin sections were baked in an oven at $60^{\circ} \mathrm{C}$ for $20 \mathrm{~min}$, and dewaxed for $5 \mathrm{~min}$ each in xylene (I, II, III), absolute ethanol (I, II), 90\% ethanol (I, II), $80 \%$ ethanol (I, II), and 70\% ethanol (I, II). The ethanol was washed away in water, and the sections were then transferred to distilled water for $2 \mathrm{~min}$ and washed with PBS for $5 \mathrm{~min}$. The sections were boiled in deionized water at $90^{\circ} \mathrm{C}$ for $15 \mathrm{~min}$. The sections were put in citrate ( $\mathrm{PH} \mathrm{6.0)}$ and cooked for $40 \mathrm{~min}$, and treated with proteinase $\mathrm{K}$ at $37^{\circ} \mathrm{C}$ for $10 \mathrm{~min}$. The sections were fixed in methanol for $10 \mathrm{~min}$ at room temperature, incubated in $70 \%$ ethanol, $85 \%$ ethanol, and $100 \%$ ethanol for $3 \mathrm{~min}$ at room temperature, and then dried naturally. The sections were added with pre-hybridization solution and incubated at $42^{\circ} \mathrm{C}$ for 1 
h, After the pre-hybridization solution was aspirated, the sections were added with probe hybridization solution and hybridized overnight at $42^{\circ} \mathrm{C}$, and washed twice with buffer for $5 \mathrm{~min}$ each time. The sections were naturally dried, and nuclei was stained with 4',6-diamidino-2-phenylindole (DAPI), washed with buffer solution, and then observed and photographed under a microscope for data analysis.

\section{Cell transfection}

The expression patterns of miR-216b in BC cell lines MDA-MB-468 and MCF-7 were determined with the help of reverse transcription quantitative polymerase chain reaction (RT-qPCR), and the cell line exhibiting the highest miR-216b expression levels was selected for subsequent cell experiments. The cells were transfected with plasmids of mimic negative control (NC), miR-216b mimic, inhibitor NC, miR-216b inhibitor, mimic NC + NC over-expression vector (oe-NC), miR-216b mimic + oe-NC, and miR-216b mimic + oe-HK2. miR-216b mimic, miR-216b inhibitor, siRNA against HK2 (siHK2), and siRNA negative control (si-NC) plasmids as shown in Supplementary Table 4 were purchased from Dharmacon Research (Lafayette, CO), with mimic $\mathrm{NC}$, inhibitor NC and siNC as negative controls (NC). Untreated cells as Blank group. MDA-MB-468 and MCF-7 cells (cell density of $3 \times 10^{5} /$ well) were seeded in 6-well plates and transfected using lipofectamine 2000 kits (Invitrogen).

\section{Dual luciferase reporter gene assay}

The binding site between miR-216b and the target gene HK2 was predicted with the help of the microRNA website (www.microRNA.org). A luciferase reporter gene assay was then performed to further verify the binding relationship between miR-216b and HK2 [49]. The pHK2-Wt plasmid was constructed with full-length 3'UTR fragment of HK2 gene inserted into the pmirGLO vector (Promega Corporation, Madison, WI). And pHK2-Mut carrier was constructed by PCR-based site-directed mutagenesis. With Renilla luciferase vector pRL-TK (TaKaRa Biotechnology Co., Ltd., Dalian, China) serving as an internal reference, the luciferase activity was measured using a Dual-Luciferase Reporter Assay System (Promega Corporation).

\section{RT-qPCR}

Total RNA content was extracted from the tissues and cells using Trizol kits (Invitrogen). The obtained RNA was reverse transcribed into cDNA using Primescript TMRT reagent kits (RRO37A, TaKaRa Biotechnology Co., Ltd.). The real time PCR was then performed with a fluorescence quantitative PCR instrument (ABI7500, Applied Biosystems,
Foster city, CA), with a reaction system consisting of $25 \mu \mathrm{l}$ of $10 \times$ PCR Buffer, $2.5 \mu \mathrm{l}$ of $25 \mathrm{~m} \mathrm{~mol} / 1 \mathrm{Mgcl}_{2}$, $1.5 \mu \mathrm{l}$ of $10 \mathrm{mmol} / 1 \mathrm{dNTP}, 0.5 \mu \mathrm{l}$ of $10 \mathrm{mmol} / 1$ Primer, $1 \mu \mathrm{l}$ of $1 \mathrm{nmol} / 1$ Probe, $0.25 \mu \mathrm{l}$ of Taq, $2.5 \mu \mathrm{l}$ of cDNA and $15 \mu \mathrm{l}$ of sterile distilled water. The reaction was repeated in 3 parallel wells. U6 was regarded as the internal reference for miR-216b and GAPDH as the internal reference for other genes (Supplementary Table 5). Relative expression of miR-216b and target genes was calculated using the $2^{-\Delta \Delta \mathrm{Ct}}$ method [50].

\section{Western blot analysis}

Total protein content was extracted from the tissues and cells using Protein lysis buffer (C0481, Sigma, St Louis, MO). Following 10\% sodium dodecyl sulfate polyacrylamide gel electrophoresis, the proteins were transferred onto a nitrocellulose membrane. The membranes were subsequently incubated with the following antibodies to HK2 (dilution ratio of $1: 1000$, ab104836, Abcam), mTOR (dilution ratio of $1: 2000$, ab2732, Abcam), p-mTOR (dilution ratio of 1:1000, ab109268, Abcam), Beclin1 (dilution ratio of 1 : 500, ab114071, Abcam), Bax (dilution ratio of 1 : 1000, ab77566, Abcam), Bcl-2 (dilution ratio of 1 : 1000, ab692, Abcam), 4EBP1 (dilution ratio of $1: 1000$, ab32130, Abcam), p-4EBP1 (dilution ratio of 1:1000, ab47365, Abcam), LC3A/B (dilution ratio of $1: 1000$, ab128025, Abcam), MMP-9 (dilution ratio of $1: 1000$, ab73734, Abcam) and $\beta$-actin (dilution ratio of 1:500, Beijing Cwbiotech Co., Ltd., Beijing, China) overnight at $4^{\circ} \mathrm{C}$. Afterwards, the horseradish peroxidase (HRP) -labeled goat anti-mouse or anti-rabbit IgG (SPA131 or SA27, dilution ratio of $1: 500$, Solarbio, Beijing, China) was incubated with the membrane at room temperature for $1.5 \mathrm{~h}$. The relative expression of the proteins was measured as previously described [51].

\section{Cell count kit-8 (CCK-8)}

The transfected MDA-MB-468 and MCF-7 cells (cell density of $2 \times 103$ cells $/ \mathrm{mL}$ ) were seeded in 96-well plates and incubated with $10 \mu \mathrm{L}$ of CCK8 reagent (C0037, Beyotime, Shanghai, China) at $37^{\circ} \mathrm{C}$ for $2 \mathrm{~h}$. Subsequently, the cell viability was detected at $0 \mathrm{~h}, 24^{\text {th }} \mathrm{h}, 48^{\text {th }}$, and $72^{\text {nd }} \mathrm{h}$ using a microplate Reader (Bio-Rad, Hercules, CA, USA) [52]. Cell growth curve was drawn with time point as $\mathrm{X}$-axis and $\mathrm{OD}$ value as Y-axis. Three parallel wells were set for each group.

\section{Scratch test}

The transfected cells (cell density of $5 \times 10^{5}$ cells/well) were inoculated in 6 well plates. Scratches were then made using a $20 \mu \mathrm{L}$ sterile pipette tip. After incubation with serum-free medium, the scratch distance was measured in the cells at $12^{\text {th }} \mathrm{h}$ and $24^{\text {th }} \mathrm{h}$ 
under an inverted microscope (Nikon) [53].

\section{Transwell assay}

After $12 \mathrm{~h}$ of transfection, the apical Transwell chamber coated with Matrigel (Sigma) was incubated with $200 \mu \mathrm{L}$ cell suspension, while the lower chamber was added with $500 \mu \mathrm{L}$ medium containing $100 \mathrm{~mL} / \mathrm{L}$ FBS (FB-1001/100; Biosera) at $37^{\circ} \mathrm{C}$ with $5 \% \mathrm{CO}_{2}$ for 24 $\mathrm{h}$. The invaded cells were observed an inverted microscope (CKX41SF, Olympus, Tokyo, Japan) [51].

\section{Flow cytometry}

After $48 \mathrm{~h}$ of transfection, cell apoptosis was analyzed using Annexin-V-FITC cell apoptosis detection kits (C1065, Beyotime). The FITC and PI fluorescence was detected with the help of a BD-Aria flow cytometer (FACSCalibur, Beckman Kurt). The cell cycle distribution was detected after staining with 1\% PI containing RNAase for $30 \mathrm{~min}$, on the flow cytometer (FACSCalibur) [54]. Three independent experiments were conducted in triplicates.

\section{Monodansylcadaverine (MDC) staining}

MDC, an acid dye, serves as a specific marker for autophagic vacuoles, and thus employed for detection and quantification of autophagy. MDA-MB-468 and MCF-7 cells were seeded in 6-well plates at a concentration of $3 \times 10^{4}$ cells/well in a 5\% CO2 incubator at $37^{\circ} \mathrm{C}$ for $24 \mathrm{~h}$. Following transfection, 50 mM MDC (HY-D1027, MedChemExpress, NJ, USA) was added to each well for 15-min incubation, and then rinsed 3 times with PBS. The nuclei were then stained with DAPI. Finally, the fluorescence was visualized under a confocal laser-scanning microscope (TCS 4D; Leica, Heidelberg, Germany).

\section{Tumor formation in nude mice}

Specific pathogen-free (SPF) nude mice (female, aged 6 weeks, weighing 18-22g) were purchased from Beijing Vital River Laboratories (Beijing, China). The procured animals were housed for 1 week under conditions of suitable temperature and humidity at a $12 \mathrm{~h} / 12 \mathrm{~h}$ light/dark cycle. The MDA-MB-468 cells transfected with mimic NC and miR-216b mimic were subsequently harvested. Briefly, the mice were subcutaneously injected with the cell suspension $(1 \times$ $10^{7}$ cells) into the right axilla. The length and width of the xenografts were measured weekly using a vernier caliper, for a total of 5 weeks. The volume $(\mathrm{V})$ was calculated as follows: $\mathrm{V}\left(\mathrm{mm}^{3}\right)=1 / 2 \times$ length $\times$ width $^{2}$. At the end of the experiment, the mice were euthanatized and the xenografts were completely excised for further analysis.

\section{Statistical analysis}

Statistical analyses were performed using the
SPSS21.0 statistical software (IBM Corp. Armonk, NY). Measurement data were expressed as mean \pm standard deviation, and tested for normal distribution and variance homogeneity. Pairwise comparison between BC and adjacent normal tissues were examined using the paired $t$ test, while skewed distributed data were tested using non-parametric Wilcoxon rank-sum test. Comparisons among multiple groups were analyzed by one-way analysis of variance (ANOVA). The least significant difference (LSD) test was used for pairwise comparison. When equal variances were not assumed, the nonparametric rank test was applied. Enumeration data were expressed by percentage or ratio, and compared using the chi-square test. Data at different time points were compared using repeated measures ANOVA. The level of statistical significance was set at $a=0.05$. A value of $p<0.05$ was regarded statistically significant.

\section{Abbreviations}

BC: breast cancer; DEGs: differentially expressed genes; EMT: epithelial-mesenchymal-transition; GEO: Gene Expression Omnibus; HK2: Hexokinase 2; KEGG: Kyoto Encyclopedia of Genes and Genomes; LSD: least significant difference; MDC: Monodansylcadaverine; mTOR: mammalian target of rapamycin; PDAC: pancreatic ductal adenocarcinoma; PPI: Protein-protein interaction; RT-qPCR: reverse transcription quantitative polymerase chain reaction.

\section{Supplementary Material}

Supplementary tables 1, 3-5.

http://www.ijbs.com/v17p2970s1.pdf

Supplementary table 2 .

http://www.ijbs.com/v17p2970s2.xls

\section{Acknowledgements}

We acknowledge and appreciate our colleagues for their valuable efforts and comments on this paper.

\section{Author Contributions}

Ting Liu designed the study, Ping Ye, Yuanyuan Ye, and Baosan Han acquired data, Ting Liu and Ping Ye helped to analyse and interpret them. Ting Liu, Yuanyuan Ye and Baosan Han wrote the manuscript. All authors read and approved the final manuscript.

\section{Funding}

This study was supported by the Shanghai Science and Technology Commission Supportive Project (No. 19441913300).

\section{Competing Interests}

The authors have declared that no competing interest exists. 


\section{References}

1. Kharman-Biz A, Gao H, Ghiasvand R, Zhao C, Zendehdel K, Dahlman-Wright K. Expression of activator protein-1 (AP-1) family members in breast cancer. BMC Cancer. 2013;13(441. doi: 10.1186/1471-2407-13-441

2. Widodo I, Dwianingsih EK, Triningsih E, Utoro T, Soeripto. Clinicopathological features of indonesian breast cancers with different molecular subtypes. Asian Pac J Cancer Prev. 2014;15(15):6109-6113. doi:

3. Oh H, Eliassen AH, Beck AH, Rosner B, Schnitt SJ, Collins LC, et al. Breast cancer risk factors in relation to estrogen receptor, progesterone receptor, insulin-like growth factor-1 receptor, and Ki67 expression in normal breast tissue. NPJ Breast Cancer. 2017;3(39. doi: 10.1038/s41523-017-0041-7

4. Bakhtavar K, Saran M, Behzadifar M, Farsi M. Magnetic resonance mammography in comparison with mammography in the discovery of multifocal, multicentric and bilateral lesions of breast cancer. Electron Physician. 2017;9(8):5083-5087. doi: 10.19082/5083

5. Dalpe G, Ngueng Feze I, Salman S, Joly Y, Hagan J, Levesque E, et al. Breast Cancer Risk Estimation and Personal Insurance: A Qualitative Study Presenting Perspectives from Canadian Patients and Decision Makers. Front Genet. 2017:8(128. doi: 10.3389/fgene.2017.00128

6. Steigedal TS, Toraskar J, Redvers RP, Valla M, Magnussen SN, Bofin AM, et al. Nephronectin is Correlated with Poor Prognosis in Breast Cancer and Promotes Metastasis via its Integrin-Binding Motifs. Neoplasia. 2018;20(4):387-400. doi: 10.1016/j.neo.2018.02.008

7. Tang J, Ahmad A, Sarkar FH. MicroRNAs in breast cancer therapy. Curr Pharm Des. 2014;20(33):5268-5274. doi:

8. He J, Zhang J, Wang D. Down-regulation of microRNA-216b inhibits IL-1beta-induced chondrocyte injury by up-regulation of Smad3. Biosci Rep. 2017;37(2):doi: 10.1042/BSR20160588

9. Zheng WW, Zhou J, Zhang CH, Liu XS. MicroRNA-216b is downregulated in hepatocellular carcinoma and inhibits HepG2 cell growth by targeting Forkhead box protein M1. Eur Rev Med Pharmacol Sci. 2016;20(12):2541-2550. doi:

10. Yao Y, Li Q, Wang H. MiR-216b suppresses colorectal cancer proliferation, migration, and invasion by targeting SRPK1. Onco Targets Ther. 2018;11(1671-1681. doi: 10.2147/OTT.S161835

11. Jana S, Sengupta S, Biswas S, Chatteriee A, Roy H, Bhattacharyya A. miR-216b suppresses breast cancer growth and metastasis by targeting SDCBP. Biochem Biophys Res Commun. 2017;482(1):126-133. doi: 10.1016/j.bbrc.2016.10.003

12. He S, Liao B, Deng Y, Su C, Tuo J, Liu J, et al. MiR-216b inhibits cell proliferation by targeting FOXM1 in cervical cancer cells and is associated with better prognosis. BMC Cancer. 2017;17(1):673. doi: 10.1186/s12885-017-3650-5

13. Gao Y, Yang Y, Yuan F, Huang J, Xu W, Mao B, et al. TNFalpha-YAP/p65-HK2 axis mediates breast cancer cell migration. Oncogenesis. 2017;6(9):e383. doi: 10.1038/oncsis.2017.83

14. Patra KC, Wang $\mathrm{Q}$, Bhaskar PT, Miller L, Wang Z, Wheaton W, et al, Hexokinase 2 is required for tumor initiation and maintenance and its systemic deletion is therapeutic in mouse models of cancer. Cancer Cell. 2013;24(2):213-228. doi: 10.1016/j.ccr.2013.06.014

15. Liu Y, Huo Y, Wang D, Tai Y, Li J, Pang D, et al. MiR-216a-5p/Hexokinase 2 axis regulates uveal melanoma growth through modulation of Warburg effect. Biochem Biophys Res Commun. 2018;501(4):885-892. doi: 10.1016/j.bbrc.2018.05.069

16. Hei C, Liu P, Yang X, Niu J, Li PA. Inhibition of mTOR signaling Confers Protection against Cerebral Ischemic Injury in Acute Hyperglycemic Rats. Int J Biol Sci. 2017;13(7):878-887. doi: 10.7150/ijbs.18976

17. Zhang Y, Zhang HE, Liu Z. MicroRNA-147 suppresses proliferation, invasion and migration through the AKT/mTOR signaling pathway in breast cancer. Oncol Lett. 2016;11(1):405-410. doi: 10.3892/ol.2015.3842

18. Xie P, Cao H, Li Y, Wang J, Cui Z. Knockdown of IncRNA CCAT2 inhibits endometrial cancer cells growth and metastasis via sponging miR-216b. Cancer Biomark. 2017;21(1):123-133. doi: 10.3233/CBM-170388

19. Mathupala SP, Ko YH, Pedersen PL. Hexokinase II: cancer's double-edged sword acting as both facilitator and gatekeeper of malignancy when bound to mitochondria. Oncogene. 2006;25(34):4777-4786. doi: 10.1038/sj.onc.1209603

20. Wolf A, Agnihotri S, Munoz D, Guha A. Developmental profile and regulation of the glycolytic enzyme hexokinase 2 in normal brain and glioblastoma multiforme. Neurobiol Dis. 2011;44(1):84-91. doi: 10.1016/j.nbd.2011.06.007

21. Ma J, Fan Y, Feng T, Chen F, Xu Z, Li S, et al. HOTAIR regulates HK2 expression by binding endogenous miR-125 and miR-143 in oesophageal squamous cell carcinoma progression. Oncotarget. 2017;8(49):86410-86422. doi: 10.18632/oncotarget.21195

22. An MX, Li S, Yao HB, Li C, Wang JM, Sun J, et al. BAG3 directly stabilizes Hexokinase 2 mRNA and promotes aerobic glycolysis in pancreatic cancer cells. J Cell Biol. 2017;216(12):4091-4105. doi: 10.1083/jcb.201701064

23. Jiao L, Zhang HL, Li DD, Yang KL, Tang J, Li X, et al. Regulation of glycolytic metabolism by autophagy in liver cancer involves selective autophagic degradation of HK2 (hexokinase 2). Autophagy. 2018;14(4):671-684. doi: 10.1080/15548627.2017.1381804

24. Vander Heiden MG. Targeting cancer metabolism: a therapeutic window opens. Nat Rev Drug Discov. 2011;10(9):671-684. doi: 10.1038/nrd3504

25. Mathupala SP, Ko YH, Pedersen PL. Hexokinase-2 bound to mitochondria: cancer's stygian link to the "Warburg Effect" and a pivotal target for effective therapy. Semin Cancer Biol. 2009:19(1):17-24 doi: 10.1016/j.semcancer.2008.11.006

26. Vo BT, Morton D, Jr., Komaragiri S, Millena AC, Leath C, Khan SA. TGF-beta effects on prostate cancer cell migration and invasion are mediated by PGE2 through activation of PI3K/AKT/mTOR pathway. Endocrinology. 2013;154(5):1768-1779. doi: 10.1210/en.2012-2074

27. Bader AG, Kang S, Zhao L, Vogt PK. Oncogenic PI3K deregulates transcription and translation. Nat Rev Cancer. 2005;5(12):921-929. doi: $10.1038 / \mathrm{nrc} 1753$

28. Yap TA, Garrett MD, Walton MI, Raynaud F, de Bono JS, Workman P. Targeting the PI3K-AKT-mTOR pathway: progress, pitfalls, and promises. Curr Opin Pharmacol. 2008;8(4):393-412. doi: 10.1016/j.coph.2008.08.004

29. Yang Y, Gao M, Lin Z, Chen L, Jin Y, Zhu G, et al. DEK promoted EMT and angiogenesis through regulating $\mathrm{PI} 3 \mathrm{~K} / \mathrm{AKT} / \mathrm{mTOR}$ pathway in triple-negative breast cancer. Oncotarget. 2017;8(58):98708-98722. doi: 10.18632/oncotarget.21864

30. Chen X, Liu X, He B, Pan Y, Sun H, Xu T, et al. MiR-216b functions as a tumor suppressor by targeting HMGB1-mediated JAK2/STAT3 signaling way in colorectal cancer. Am J Cancer Res. 2017;7(10):2051-2069. doi:

31. Huang G, Pan J, Ye Z, Fang B, Cheng W, Cao Z. Overexpression of miR-216b sensitizes NSCLC cells to cisplatin-induced apoptosis by targeting c-Jun. Oncotarget. 2017;8(61):104206-104215. doi: 10.18632/oncotarget.22171

32. Ni H, Rui Q, Zhu X, Yu Z, Gao R, Liu H. Antihypertensive drug use and breast cancer risk: a meta-analysis of observational studies. Oncotarget. 2017;8(37):62545-62560. doi: 10.18632/oncotarget.19117

33. Kato T, Park JH, Kiyotani K, Ikeda Y, Miyoshi Y, Nakamura Y. Integrated analysis of somatic mutations and immune microenvironment of multiple regions in breast cancers. Oncotarget. 2017;8(37):62029-62038. doi: 10.18632/oncotarget.18790

34. Deng M, Tang H, Zhou $\mathrm{Y}$, Zhou M, Xiong $\mathrm{W}$, Zheng $\mathrm{Y}$, et al. miR-216b suppresses tumor growth and invasion by targeting KRAS in nasopharyngeal carcinoma. J Cell Sci. 2011;124(Pt 17):2997-3005. doi: 10.1242/jcs.085050

35. Chen Z, Gao S, Wang D, Song D, Feng Y. Colorectal cancer cells are resistant to anti-EGFR monoclonal antibody through adapted autophagy. Am J Transl Res. 2016;8(2):1190-1196. doi:

36. Xu Z, Bu Y, Chitnis N, Koumenis C, Fuchs SY, Diehl JA. miR-216b regulation of c-Jun mediates GADD153/CHOP-dependent apoptosis. Nat Commun. 2016:7(11422. doi: $10.1038 /$ ncomms11422

37. Sato-Tadano A, Suzuki T, Amari M, Takagi K, Miki Y, Tamaki K, et al. Hexokinase II in breast carcinoma: a potent prognostic factor associated with hypoxia-inducible factor-1alpha and Ki-67. Cancer Sci. 2013;104(10):1380-1388. doi: $10.1111 /$ cas. 12238

38. Jin F, Wang $Y$, Zhu Y, Li S, Liu $Y$, Chen $C$, et al. The miR-125a/HK2 axis regulates cancer cell energy metabolism reprogramming in hepatocellular carcinoma. Sci Rep. 2017;7(1):3089. doi: 10.1038/s41598-017-03407-3

39. Wu J, Hu L, Wu F, Zou L, He T. Poor prognosis of hexokinase 2 overexpression in solid tumors of digestive system: a meta-analysis. Oncotarget. 2017;8(19):32332-32344. doi: 10.18632/oncotarget.15974

40. Anderson M, Marayati R, Moffitt R, Yeh JJ. Hexokinase 2 promotes tumor growth and metastasis by regulating lactate production in pancreatic cancer. Oncotarget. 2017;8(34):56081-56094. doi: 10.18632/oncotarget.9760

41. Lee SY, Tsai WC, Lin JC, Ahmetaj-Shala B, Huang SF, Chang WL, et al. Astragaloside II promotes intestinal epithelial repair by enhancing L-arginine uptake and activating the mTOR pathway. Sci Rep. 2017;7(1):12302. doi: 10.1038/s41598-017-12435-y

42. Fu YF, Liu X, Gao M, Zhang YN, Liu J. Endoplasmic reticulum stress induces autophagy and apoptosis while inhibiting proliferation and drug resistance in multiple myeloma through the PI3K/Akt/mTOR signaling pathway. Oncotarget. 2017;8(37):61093-61106. doi: 10.18632/oncotarget.17862

43. Karlsson E, Perez-Tenorio G, Amin R, Bostner J, Skoog L, Fornander T, et al. The mTOR effectors $4 \mathrm{EBP} 1$ and $\mathrm{S} 6 \mathrm{~K} 2$ are frequently coexpressed, and associated with a poor prognosis and endocrine resistance in breast cancer: a retrospective study including patients from the randomised Stockholm tamoxifen trials. Breast Cancer Res. 2013;15(5):R96. doi: 10.1186/bcr3557

44. Tang Z, Kang B, Li C, Chen T, Zhang Z. GEPIA2: an enhanced web server for large-scale expression profiling and interactive analysis. Nucleic Acids Res. 2019;47(W1):W556-W560. doi: 10.1093/nar/gkz430

45. Pinero J, Bravo A, Queralt-Rosinach N, Gutierrez-Sacristan A, Deu-Pons J, Centeno $\mathrm{E}$, et al. DisGeNET: a comprehensive platform integrating information on human disease-associated genes and variants. Nucleic Acids Res. 2017;45(D1):D833-D839. doi: 10.1093/nar/gkw943

46. Pinero J, Queralt-Rosinach N, Bravo A, Deu-Pons J, Bauer-Mehren A, Baron $\mathrm{M}$, et al. DisGeNET: a discovery platform for the dynamical exploration of human diseases and their genes. Database (Oxford). 2015;2015(bav028. doi: 10.1093/database/bav028

47. Heo MH, Cho YJ, Kim HK, Kim JY, Park YH. Isolated pachymeningeal metastasis from breast cancer: Clinical features and prognostic factors. Breast. 2017;35(109-114. doi: 10.1016/j.breast.2017.07.006

48. Jin YP, Hu YP, Wu XS, Wu YS, Ye YY, Li HF, et al. miR-143-3p targeting of ITGA6 suppresses tumour growth and angiogenesis by downregulating PLGF expression via the PI3K/AKT pathway in gallbladder carcinoma. Cell Death Dis. 2018;9(2):182. doi: 10.1038/s41419-017-0258-2

49. Rice SJ, Lai SC, Wood LW, Helsley KR, Runkle EA, Winslow MM, et al. MicroRNA-33a mediates the regulation of high mobility group AT-hook 2 
gene (HMGA2) by thyroid transcription factor 1 (TTF-1/NKX2-1). J Biol Chem. 2013;288(23):16348-16360. doi: 10.1074/jbc.M113.474643

50. Livak KJ, Schmittgen TD. Analysis of relative gene expression data using real-time quantitative PCR and the 2(-Delta Delta C(T)) Method. Methods. 2001;25(4):402-408. doi: 10.1006/meth.2001.1262

51. Ye YY, Mei JW, Xiang SS, Li HF, Ma Q, Song XL, et al. MicroRNA-30a-5p inhibits gallbladder cancer cell proliferation, migration and metastasis by targeting E2F7. Cell Death Dis. 2018;9(3):410. doi: 10.1038/s41419-018-0444-x

52. Cui S, Liao X, Ye C, Yin X, Liu M, Hong Y, et al. ING5 suppresses breast cancer progression and is regulated by miR-24. Mol Cancer. 2017;16(1):89. doi: 10.1186/s12943-017-0658-z

53. Sharma P, Kumar S. Metformin inhibits human breast cancer cell growth by promoting apoptosis via a ROS-independent pathway involving mitochondrial dysfunction: pivotal role of superoxide dismutase (SOD). Cell Oncol (Dordr). 2018;41(6):637-650. doi: 10.1007/s13402-018-0398-0

54. Yu X, Luo A, Liu Y, Wang S, Li Y, Shi W, et al. MiR-214 increases the sensitivity of breast cancer cells to tamoxifen and fulvestrant through inhibition of autophagy. Mol Cancer. 2015;14(208. doi: $10.1186 /$ s12943-015-0480-4 